Revue musicale OICRM

\title{
Claude Debussy. Modèle à suivre ou à éviter ? La réception du maître français chez Alfredo Casella
}

\section{Justine Comtois}

Volume 2, numéro 1, 2014

La réception de Debussy au XXe siècle. Incidences, influences et autorité

URI : https://id.erudit.org/iderudit/1055848ar

DOI : https://doi.org/10.7202/1055848ar

Aller au sommaire du numéro

Éditeur(s)

OICRM

ISSN

2368-7061 (numérique)

Découvrir la revue

Citer cet article

Comtois, J. (2014). Claude Debussy. Modèle à suivre ou à éviter ? La réception du maître français chez Alfredo Casella. Revue musicale OICRM, 2(1), 101-115. https://doi.org/10.7202/1055848ar
Résumé de l'article

Tout au long de sa carrière de compositeur, Alfredo Casella (1883-1947) semble faire un cas particulier du maître français Claude Debussy. Lors de sa période de formation en France (1896-1915) Debussy est une source d'inspiration majeure pour le jeune compositeur en quête d'un style qui lui soit personnel. De retour en Italie, Casella encourage les jeunes compositeurs à s'inspirer des techniques européennes, incluant celles de Debussy, pour moderniser la musique instrumentale italienne. Cependant, avec la mise de l'avant du passé musical italien, pour la création d'un art national, Casella adopte progressivement une position différente, voire diamétralement opposée, face à la musique de Debussy. Le compositeur français n'est alors plus un modèle, ni même un "rêve pour les générations futures ", mais plutôt, si l'on en croit Casella, l'auteur d'un langage tout à fait incompatible avec «l'esprit profondément italien ». Nous proposons une étude de nature critique, historique et esthétique d'oeuvres de Casella datant de 1902 à 1930. Nous tenterons d'établir un lien méthodologique entre les affirmations et les opinions dans les écrits de Casella et leur application dans ses compositions par l'entremise de l'argumentation développée par le musicien. 


\title{
Claude Debussy. Modèle à suivre ou à éviter ? La réception du maître français chez Alfredo Casella
}

\author{
Justine Comtois
}

\begin{abstract}
Résumé
Tout au long de sa carrière de compositeur, Alfredo Casella (1883-1947) semble faire un cas particulier du maître français Claude Debussy. Lors de sa période de formation en France (1896-1915) Debussy est une source d'inspiration majeure pour le jeune compositeur en quête d'un style qui lui soit personnel. De retour en Italie, Casella encourage les jeunes compositeurs à s'inspirer des techniques européennes, incluant celles de Debussy, pour moderniser la musique instrumentale italienne. Cependant, avec la mise de l'avant du passé musical italien, pour la création d'un art national, Casella adopte progressivement une position différente, voire diamétralement opposée, face à la musique de Debussy. Le compositeur français n'est alors plus un modèle, ni même un "rêve pour les générations futures ", mais plutôt, si l'on en croit Casella, l'auteur d'un langage tout à fait incompatible avec "l'esprit profondément italien ». Nous proposons une étude de nature critique, historique et esthétique d'œuvres de Casella datant de 1902 à 1930. Nous tenterons d'établir un lien méthodologique entre les affirmations et les opinions dans les écrits de Casella et leur application dans ses compositions par l'entremise de l'argumentation développée par le musicien.
\end{abstract}

Mots clés : Casella ; Debussy ; impressionnisme ; influence ; Italie.

\begin{abstract}
Throughout his career, Alfredo Casella (1883-1947) seemed to make a particular case of French master Claude Debussy. During his training period in France (18961915), Debussy was a major source of inspiration to the young composer seeking his own personal style. Back in Italy, Casella encouraged young composers to inspire themselves of European techniques, including those of Debussy, to modernize the Italian instrumental music. However, with the promotion of the Italian musical past, aiming the creation of a national art, Casella gradually adopted a different position, event diametrically opposed, regarding Debussy's music. Then the French composer is not considered as a model anymore, nor even, a "dream for the future generations," but rather, according to Casella, the author of a language entirely incompatible with "the profoundly Italian spirit." We propose a critical, historical and aesthetical study of Casella's works from 1902 to 1930 . We shall try establishing a methodological link between the assertions and opinions in Casella's writings and their application in his musical works, by the means of the argumentation developed by the musician.
\end{abstract}

Keywords: Casella; Debussy; Impressionism; influence; Italy. 
Il est incontestable que l'œuvre de Claude Debussy a changé le cours de l'évolution musicale européenne et l'Italie n'a pas échappé à son influence. À la fin du XIX ${ }^{\mathrm{e}}$ siècle, l'œuvre de Debussy est pratiquement inconnue dans la péninsule. L'ouverture du pays aux autres cultures est alors encore très limitée et la grande majorité des compositeurs italiens reçoit son éducation musicale en Italie même plutôt que de s'exiler à l'étranger pour poursuivre sa formation. La musique nationale est fortement dominée par la musique opératique et les institutions musicales italiennes accusent un sérieux retard par rapport au reste de l'Europe en ce qui a trait à l'enseignement des musiques instrumentales. Seule une petite minorité de compositeurs italiens préfère poursuivre sa formation musicale à l'étranger, choisissant principalement l'Allemagne, comme c'est le cas, par exemple, pour Ferruccio Busoni, Franco Alfano, Carlo Perinello, Vincenzo Davico, Vincenzo Tommasini et Ottorino Respighi. Seuls Franco Alfano et Alfredo Casella poursuivront leur éducation musicale en France.

C'est en 1896, alors qu'il n'est âgé que de 13 ans, qu'Alfredo Casella quitte l'Italie pour poursuivre sa formation musicale au Conservatoire de Paris. Il passera 19 années dans la capitale française au cours desquelles il mènera la vie d'un authentique musicien français, prenant part aux activités des salons parisiens les plus en vue ${ }^{1}$, puisant ses inspirations dans les œuvres nouvelles des musiciens qu'il côtoie et prenant une part active dans les débats qui animent alors la vie musicale française. Casella est en effet très présent dans la presse musicale française ${ }^{2}$. Dans ses nombreux articles, Casella prend certes position dans les débats musicaux de l'époque (autour de $\mathrm{Mahler}^{3}$ et de Stravinsky ${ }^{4}$ notamment), mais la question de la latinité revient également de manière récurrente. Avec cette latinité, Casella cite souvent l'œuvre de Debussy :

[Je] me souviens d'avoir assisté récemment à un concert dominical, sur le programme duquel voisinait la symphonie de Franck, le Prélude à l'après-midi d'un faune de Debussy et le Concerto en ut mineur de Saint-Saëns, et d'y avoir constaté en toute sincérité que Saint-Saëns et Debussy offraient entre eux un lien ethnique, alors qu'il était matériellement impossible de trouver la moindre parenté entre l'un d'eux et Franck. J'eus ce jour-là la perception nette et définitive que Saint-Saëns et Debussy appartenaient tous deux (malgrél'abîme qui sépare leurs personnalités) à la grande famille gréco-latine, à laquelle il est impossible de rattacher Franck malgré sa naturalisation française et les

1 Casella se produit régulièrement aux soirées organisées par Sophie et Paul Clémenceau.

2 Casella collabore notamment aux journaux suivants : Le monde musical, L'homme libre, Montjoie!, Revue musicale sIM et La revue musicale.

3 «Mise au point. À propos des modifications apportées par M. G. Mahler dans l'instrumentation des Symphonies de Beethoven ", Le monde musical (30 janvier 1910), p. 22-24. / "Gustav Mahler ", Le monde musical (1911), p. 154. / "Gustav Mahler », Le guide du concert (1911) , p. 425-26. / "Gustav Mahler et sa Deuxième Symphonie ", Revue musicale SIM (15 avril 1910), p. 238-250. / " La Cinquième Symphonie de Mahler ", Le monde musical (30 janvier 1911), p. 26. / "La Huitième Symphonie de Gustav Mahler. Notes sur l'Exécution », Le monde musical (1910), p. 259-260.

4 «Le Sacre du printemps aux Concerts Monteux », L’homme libre (6 avril 1914), p. 2. / « Ce qu'est la musique polyharmonique », Montjoie! (juin 1914). 
efforts de M. d'Indy. Et devant la maladresse flagrante (quoique géniale) et le lourd mysticisme nordique et brumeux de la symphonie franckiste je goûtai avec joie, tant chez Saint-Saëns que chez Debussy, le charme de cette clarté, cette netteté de forme, cette perfection de réalisations communes à tous les grands esprits de la civilisation à laquelle nous nous honorons d'appartenir (Casella, « Saint-Saëns et les jeunes »).

Nous proposons une étude de la réception de Debussy dans les œuvres et les écrits caselliens. Non seulement Casella s'inspire-t-il de Debussy dans ses créations musicales, mais de tous les compositeurs italiens de sa génération, il est celui qui a le plus écrit à propos de Debussy. Il a également côtoyé le compositeur français, surtout au début des années 1910, a interprété et dirigé plusieurs de ses œuvres dont les trois Nocturnes et Ibéria ${ }^{5}$.

\section{Casella et L'Influence Debussyste}

\section{Rencontre avec Debussy et son cuvre}

L'œuvre de Debussy fut révélée à Casella pour la première fois en 1898 aux Concerts Lamoureux. Il y entend le Prélude à l'après-midi d'un faune qui, comme il en témoigne dans ses mémoires, constitue pour lui la découverte d'un nouvel univers (Casella 1941, p. 78). En 1916, il écrira que « ce chef-d'œuvre de Debussy intitulé Prélude à l'après-midi d'un faune me semble souvent être une musique d'un italien méridional, lequel eut ébauché dans ce poème les visions païennes suggérées par notre île de Polyphème ${ }^{6}$ ". Casella écrit encore que Nuages et Fêtes lui donnent l'impression de connaître un monde nouveau, provoquant en lui une ivresse infinie et l'élargissement de sa sensibilité musicale (ibid., p. 84).

Lors de sa période française (1896 à 1915), Casella s'inspire fortement des œuvres de compositeurs français, comme Fauré ${ }^{7}$ ou encore Ravel - son grand $\mathrm{ami}^{8}$ - mais également de l'œuvre de Debussy, qui occupe une place toute particulière tant dans l'œuvre que dans les écrits caselliens.

Casella compositeur est un véritable caméléon. Il ne s'inspire pas seulement d'autres compositeurs, mais va généralement jusqu'à pasticher leurs œuvres, et Debussy n'échappe pas à son attention. Le traitement de la voix chez Casella, notamment l'utilisation d'un ambitus réduit, pourrait nous faire confondre les œuvres du jeune Italien à bien des mélodies françaises de l'époque. Mais le traitement quasi orchestral

$5 \quad$ Notamment aux concerts Hasselmans en 1912.

6 "Quel capolavoro debussiano intitolato Prélude à l'après-midi d'un faune mi sembra spesso musica di un italiano meridionale, il quale avesse abbozzato in questo poema le visioni pagane suggerite a noi tutti dall'isola di Polifemo » (Casella janvier 1916, p. 2).

$7 \quad$ Fauré est le professeur de composition de Casella au Conservatoire.

8 Casella et Ravel se rencontrent dans la classe de Gabriel Fauré au Conservatoire. Ils demeurent par la suite de grands amis et fréquentent les mêmes salons. Ils s'associent également en 1913 pour la composition de la deuxième série de À la manière de... 
$\mathrm{du}$ piano donne aux lyriques de Casella un caractère moins intimiste que celui des mélodies de l'époque, conçues pour être jouées dans les salons. Nous ne proposons pas ici une analyse exhaustive de l'œuvre de Casella, mais plutôt quelques exemples de ces inspirations debussystes. Les premières mélodies caselliennes, Nuageries (1902-1903) par exemple, s'inspirent largement de l'atmosphère évanescente, des effets d'hallucinations sinistres et floues grâce aux notes graves du piano et à l'ambitus restreint de la voix, des dégoulinades debussystes propres à Nuages. Nous retrouvons quelques références aux Nocturnes de Debussy, ou plutôt de ce "Magister Humidus ", tel que 1'avait surnommé le compositeur Alberto Savinio ${ }^{9}$.
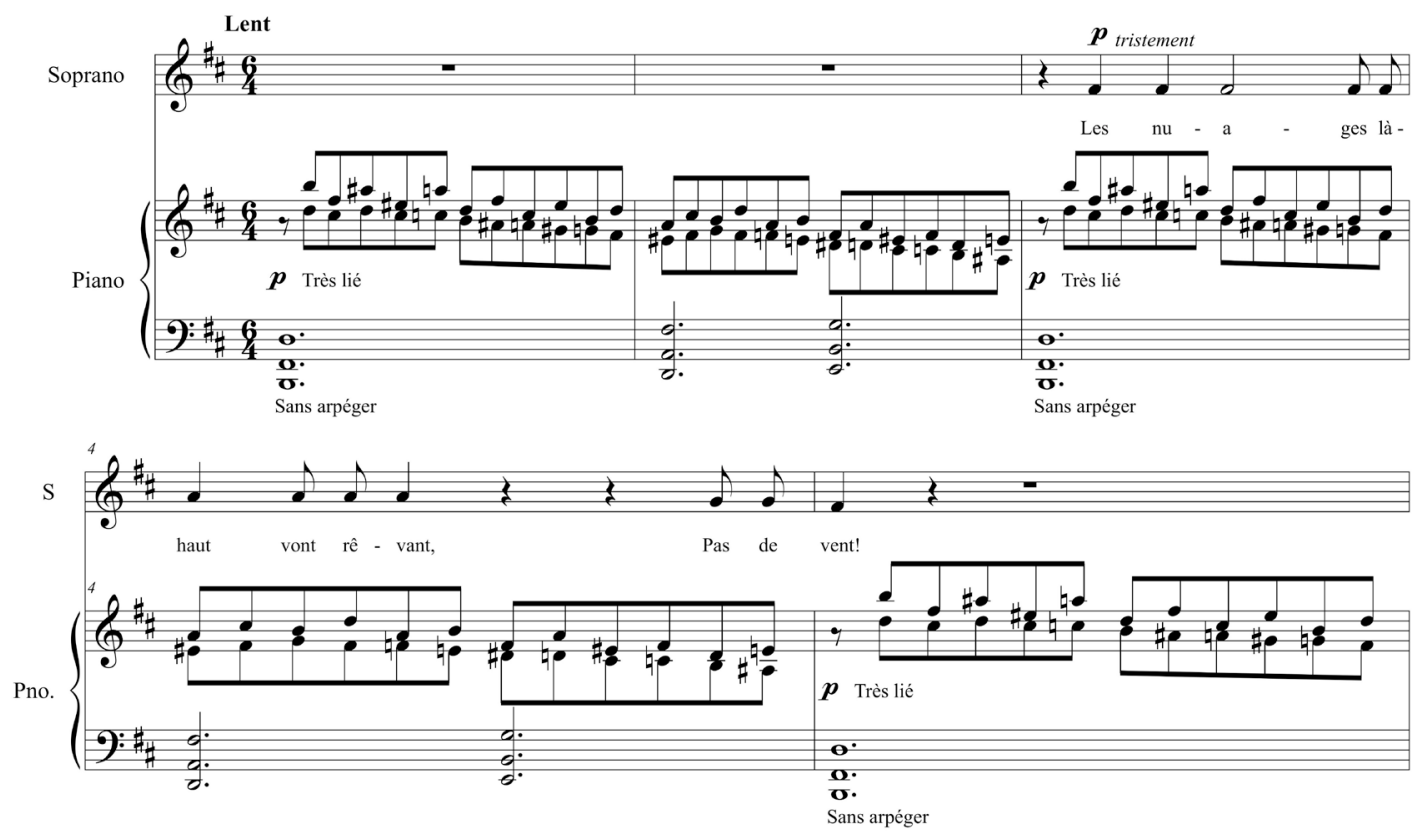

Figure 1 : Alfredo Casella, Nuageries, op. 2 (1902-1903).

Dans sa Toccata $^{10}$ pour piano solo datant de 1904, Casella fait une fois de plus appel à l'œuvre debussyste, puisant cette fois-ci son inspiration de la " toccata » finale tirée de Pour le piano ${ }^{11}$. Dès ses tous premiers contacts avec l'œuvre debussyste, Casella voue une véritable admiration pour les recherches harmoniques du maître français. La Toccata contient les premières expérimentations harmoniques de Casella, qui y fait ses premières armes dans l'utilisation simultanée de différentes gammes, une technique plus largement utilisée dans les œuvres ultérieures.

9 Pour cette appellation, Savinio s'inspire de l'appellation de " Magister Claudius " que Gabriele d'Annunzio attribuat à Debussy dans le Martyre de Saint-Sébastien (Spampinato 2011, p. 110).

10 Cette œuvre est dédiée à Édouard Risler (1873-1929).

11 On y retrouve une clarté et une limpidité de l'écriture pianistique qui rappellent également certaines œuvres de Ravel dont Le menuet antique et la Sonatine, bien que l'écriture pianistique soit complexe et qu'elle requiert une grande virtuosité. 


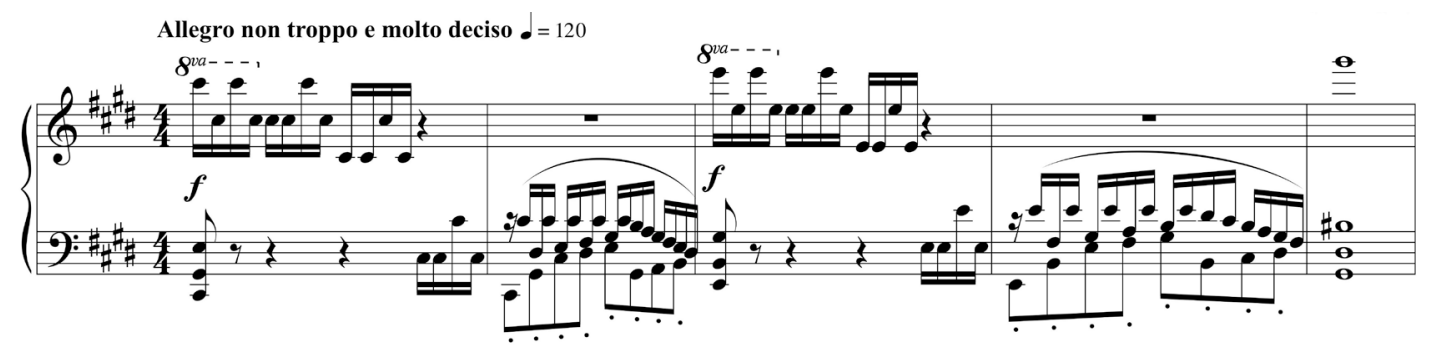

Figure 2: Alfredo Casella, Toccata, op. 6 (1904).

En 1915, il crée également les Pagine di guerra, quatre pièces pour piano quatre mains rendant hommage aux soldats alliés de la Triple-Entente ${ }^{12}$. L'origine de ces "Pages de guerre ", dont le sous-titre est "Quattro "films " musicali per pianoforte à quattro mani ${ }^{13}$ ", est incertaine : peut-être étaient-elles destinées à accompagner un film muet, documentaire sur la guerre, ou au contraire, Casella a-t-il été inspiré par les images cinématographiques que l'on présentait fréquemment aux civils durant la guerre. Aucun élément ne permet d'apporter une réponse précise. Roberto Calabretto suggère également que Casella aurait pu être aidé par ses amis Erik Satie et Florent Schmitt, qui avaient eux-mêmes été parmi les premiers à créer des musiques pour le cinéma muet et par Émile Vuillermoz, grand amateur de cinéma (Calabretto 2001, p. 94).

Respectivement intitulées "Nel Belgio. Sfilata di artiglieria pesante tedesca ", "In Francia. Davanti alle rovine della cattedrale di Reims ", "In Russia. Carica di cavalleria cosacca» et "In Alsazia. Croci di legno... " ${ }^{14}$, les quatre courts mouvements de l'œuvre ont définitivement un caractère descriptif, voire même programmatique en rapport direct avec la guerre. D'abord créées pour piano à quatre mains, Casella les orchestre en 1918 et y ajoute un cinquième mouvement intitulé "Nell'Adriatico. Corazzate italiane in crochera "

La partie intitulée "En France. Devant les ruines de la cathédrale de Reims » fait référence au bombardement de la cathédrale en 1914, une tragédie qui entraîne une profonde désolation et inspire plusieurs compositeurs, parmi lesquels nos deux principaux intéressés. Notons que le bombardement de cette Cathédrale entraîne la plus profonde désolation et nombreux sont les compositeurs français à consacrer une œuvre ou encore à organiser diverses manifestations pour dénoncer ce triste événement ${ }^{16}$. La Cathédrale de Reims devient 1'une des métaphores les plus représentatives des destructions causées par la guerre (Watkins 2003, p. 28). Avec ce deuxième

12 Alliance militaire de la France, du Royaume-Uni et de la Russie impériale. La Triple-Entente était opposée à la Triplice (Empires allemand et austro-hongrois et le royaume d'Italie).

13 "Quatre films musicaux pour piano à quatre mains ».

14 «En Belgique. Défilé d'artillerie lourde allemande», «En France. Devant les ruines de la cathédrale de Reims », «En Russie. Charge de cavalerie cosaque » et « En Alsace. Croix de bois... ».

15 "Sur l'Adriatique. Cuirassés italiens en croisière ».

16 Mentionnons La cathédrale victorieuse d'Amédée Reuschel (1915) et le concert hommage organisé par Gabriel Pierné le 28 mars 1915 à la salle Gaveau (Buch 2009, p. 136). Notons également le « Au-dessus de la mêlée » de Romain Rolland (29 août 1914), Le vent dans les ruines de Jacques Ibert. Auguste Rodin a également rédigé une lettre, datant du 30 septembre 1914, pour supporter Romain Rolland (Watkins 2003, p. 28-29). Notons finalement les nombreuses campagnes de dénonciation menées par le chansonnier Émile Jacques-Delcroze. 
mouvement, Casella rend non seulement hommage aux alliés de l'Italie, mais s'inscrit également dans l'actualité et s'associe à la lignée des artistes français de son époque. En détruisant la Cathédrale de Reims, les Allemands portaient atteinte à tout un pan de l'histoire et à un symbole plus que significatif de la culture française ${ }^{17}$. L'œuvre de Casella est empreinte d'un certain impressionnisme rappelant La cathédrale engloutie de Debussy. D'autres passages rappellent également, par leur tessiture et l'utilisation d'accords parallèles, En blanc et noir de Debussy.

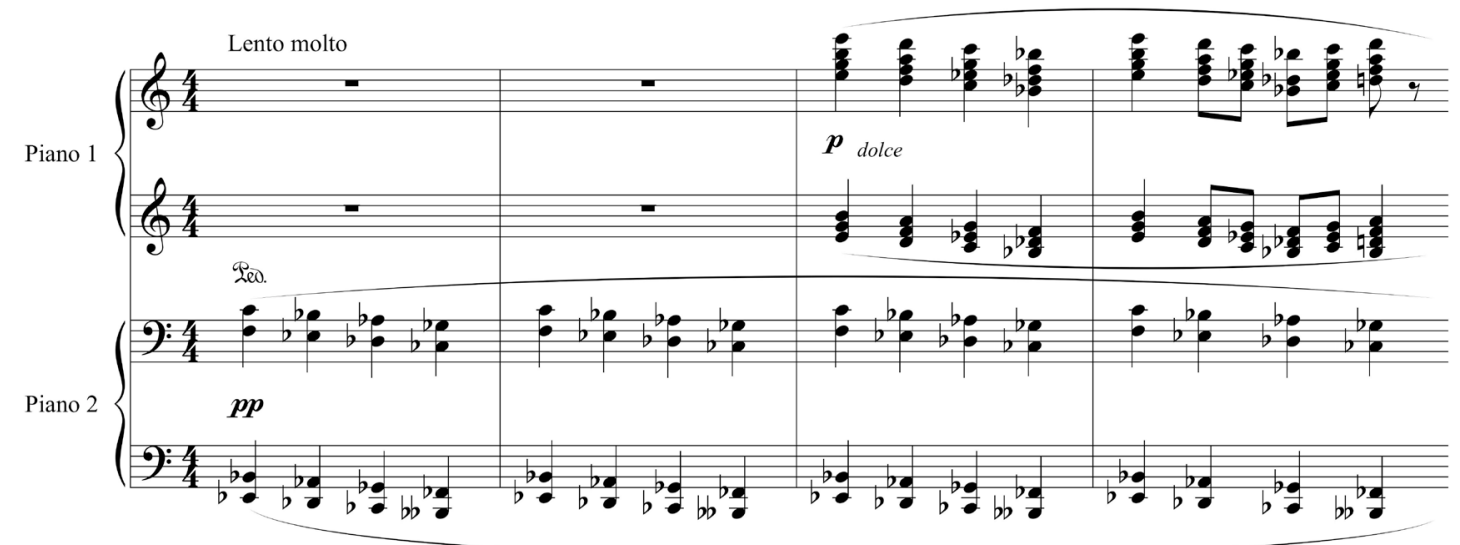

Figure 3: Alfredo Casella, Pagine di guerra, op. 25, "In Francia. Davanti alle rovine della cattedrale di Reims» (1915).

Également, dans la dernière partie, Casella n'hésite pas à s'inspirer du compositeur français et à citer La Marseillaise, comme Debussy l'avait fait dans Feux d'artifice avec cette atmosphère on ne peut plus vague et impressionniste, à une époque où il affirmait s'être pourtant détaché de cette esthétique. En effet, Casella précise dans ses mémoires que l'environnement français n'eut sur lui qu'une très faible influence. Il ne trouve d'autre réponse à ceci que la force de sa nature italienne profondément anti-impressionniste (Casella 1941, p. 114). Par anti-impressionniste, Casella entend une musique claire, exempte de nébulosité, robuste, s'inscrivant dans la lignée de la tradition musicale italienne.

17 Cette cathédrale a servi au couronnement des rois français (dont celui de Charles VII avec, à ses côtés, Jeanne d'Arc). De plus, Reims était une ville archiépiscopale depuis le huitième siècle. 


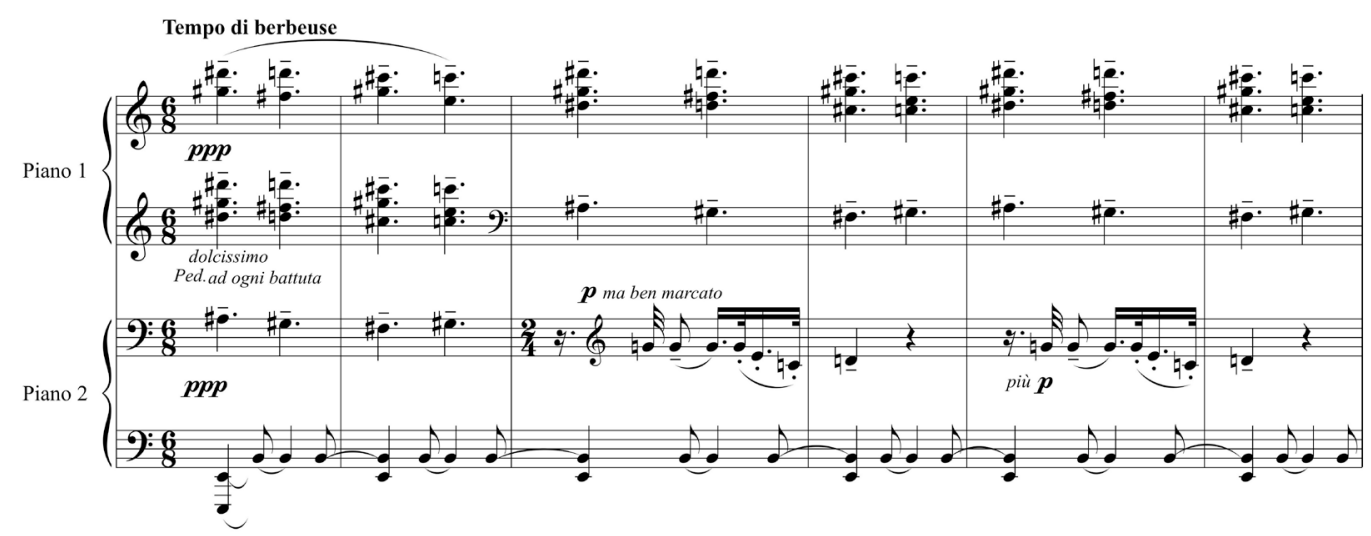

Figure 4: Alfredo Casella, Pagine di guerra, op. 25, "En Alsace. Croix de bois... » (1915).

Dans ces deux "mouvements français ", Casella incorpore plusieurs mélodies italiennes à tendance folklorique, mariant encore une fois nationalisme et influences étrangères. Il y fait également l'usage du mode phrygien, généralement associé à la guerre, représentant ainsi deux alliés : les Français et les Italiens. Il s'agit là davantage d'une musique pour les alliés que d'une musique purement consacrée à l'honneur de la patrie italienne.

Les dons de pasticheur de Casella rejoignent ses dons de caricaturiste avec la création en $1911^{18}$ et en 1914, de deux recueils d'œuvres pour piano rendant hommage à quelques compositeurs modernes, intitulés $\grave{A}$ la manière de. Le second recueil sera créé en collaboration avec Maurice Ravel ${ }^{19}$. Avec cette œuvre, Casella s'inscrit dans une tendance très populaire à l'époque dans la littérature et la musique françaises : celle du pastiche. Ces œuvres, jusqu'à leurs titres, sont emplies de clichés et de fines observations musicales rappelant à merveille les œuvres des compositeurs qu'elles imitent. La caricature caustique consacrée à Debussy, intitulée "Entr'acte pour un drame en préparation ", rappelle l'œuvre du maître français par ses clichés, ses nuances pianissimo, son atmosphère diffuse et l'utilisation presqu'abusive des accords parallèles.

18 Le premier recueil (1911) comprend les mouvements suivants : Wagner, «Einleitung des 3. Aufzuges "; Fauré, " Romance sans paroles »; Brahms, "Intermezzo »; Debussy, " Entr'acte pour un drame en préparation »; Strauss, "Symphonia molestica »; Franck, « Aria ».

19 Ce second recueil (1914) comprend les mouvements suivants : Borodine, «Valse » (Ravel) ; D'Indy, "Prélude à l'après-midi d'un Ascète » (Casella) ; Chabrier, " Paraphrase sur un air de Gounod (Faust IIème acte) » (Ravel) ; Ravel, «Almanzor ou le mariage d'Adelaïde » (Casella). 

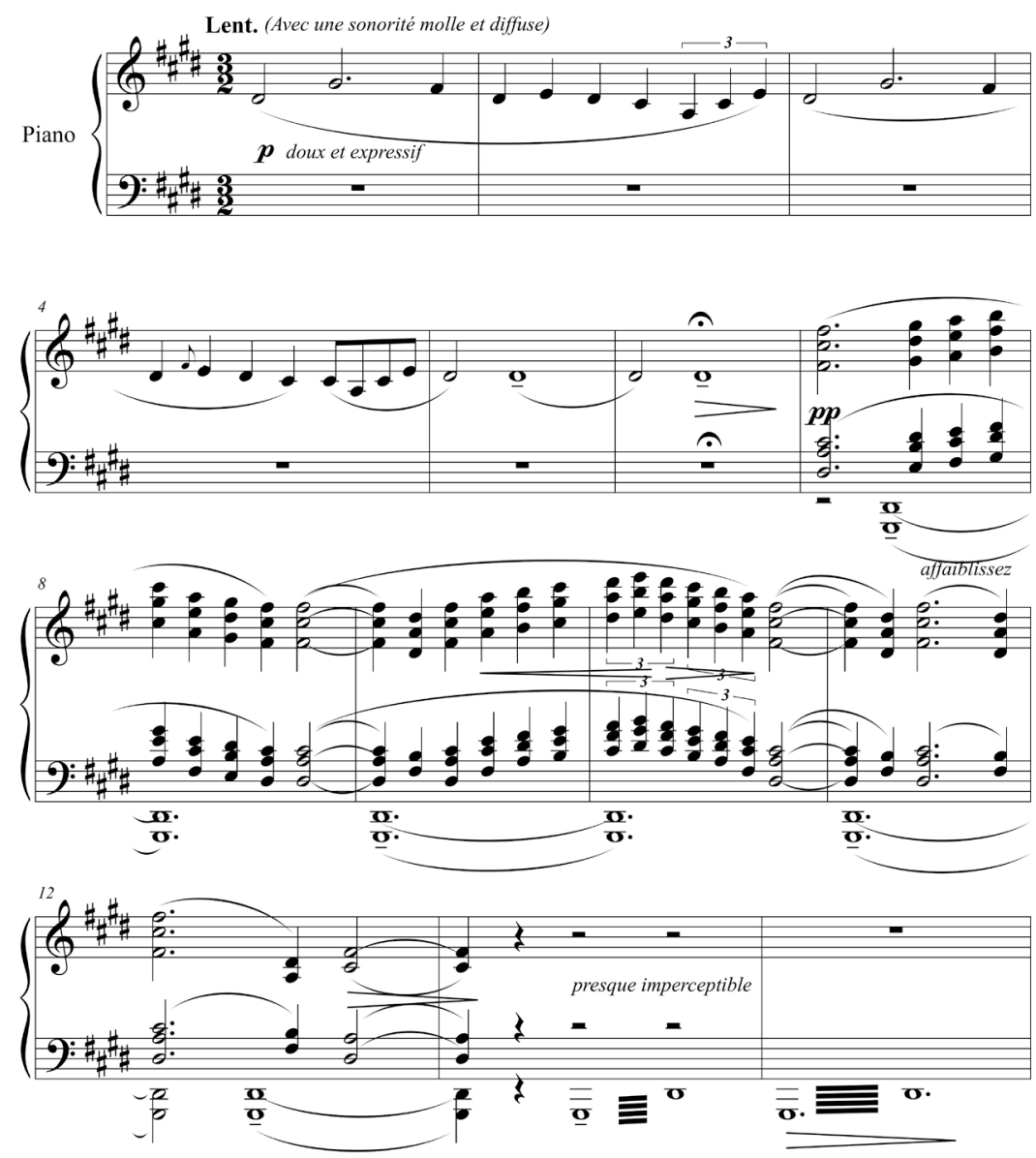

Figure 5 : Alfredo Casella, À la manière de... Debussy, "Entr'acte pour un drame en préparation ", op. 17 (1911).

Debussy constitue, aux yeux de Casella, l'une des principales révélations du $\mathrm{XIX}^{e}$ siècle. Comme en témoignent ses œuvres des années 1910 - Nove Pezzi, Notte di maggio ou encore Pagine di guerra - et ses œuvres ultérieures, les innovations harmoniques et la recherche de nouvelles sonorités sont le point d'honneur de Casella. Debussy sera d'ailleurs l'objet de plusieurs de ses études et sera souvent cité en exemple dans plusieurs de ses écrits théoriques (Casella 1916 ; $1918 ; 1920 ; 1922$; $1926 ; 1930)$.

\section{Nouvelles recherches musicales}

Dans les années 1910, Casella démontre une véritable obsession pour ce qu'il définit comme la polyharmonie, c'est-à-dire la possibilité de superposer, de manière simultanée, six à douze sons différents et de les regrouper en deux ou trois divisions distinctes se réclamant d'une tonalité différente. Il considère cette polyharmonie comme un puissant moyen d'analyse et de simplification harmonique, mais également et surtout comme une manière d'obtenir de nouvelles sonorités. Sa conception de l'harmonie s'oppose à la vision debussyste. Casella se définit par rapport à Debussy 
comme un harmoniste plutôt que comme un mélodiste. Il estime que des trois éléments qui composent la musique (harmonie, mélodie, rythme), l'harmonie est le plus important (Casella 1915, p. 1). Avec cette affirmation, Casella accentuera sa volonté d'indépendance face à la tradition lyrique italienne, où la mélodie domine.

Casella précise sa position en affirmant que Debussy a apporté ce qu'il définit comme la polymodalité horizontale (ou mélodique), par opposition à la polymodalité ou à la polytonalité harmonique, accordique, verticale, en simultanéité ce qui a eu pour effet d'étendre les combinaisons sonores à l'infini. Il allait donc de soi, selon lui, que la génération suivante ait pour réflexe d'introduire ces récentes découvertes en simultanéité, pour en arriver, entre 1910 et 1914, à la création de la polytonalité (Casella 1923, p. 69). Plus tard, dans les années 1930, cette polytonalité sera pour Casella la seule solution possible permettant d'endiguer la disparition de l'harmonie, conséquence des excès chromatiques du romantisme wagnérien et des agrégats debussystes, qu'il considère comme dépassés.

Cette nouvelle primauté accordée aux recherches harmoniques est accompagnée par la volonté croissante de Casella de se rapprocher de ce qu'il définit comme l'esprit italien. Toutefois, précisons que le nationalisme de Casella a ceci de particulier qu'il est toujours associé à un intérêt marqué pour les autres cultures. Cet intérêt se traduit d'ailleurs fort bien dans ses œuvres musicales dans lesquelles on retrouve des influences russes, roumaines, françaises espagnoles et quelques fois américaines.

\section{PREMIÈRES EXPRESSIONS DU NATIONALISME}

Casella s'éveille au nationalisme en 1909, alors qu'il vit encore à Paris. Il entend pour la première fois Ibéria non pas de Debussy, mais d'Isaac Albéniz dont il devient « fou » (Casella 1930, 496). C'est cette œuvre qui, comme il le révèle dans un article autobiographique (ibid.), stimulera en lui son sentiment d'appartenance à son pays d'origine et le désir d'accomplir en Italie ce qu'Albéniz avait fait en Espagne ${ }^{20}$. Dans un essai sur sa musique et ses propres influences, il écrit qu'il lui semble considérable pour Albéniz d'être parvenu à écrire cette musique résolument plastique et ainsi franchement anti-impressionniste à Paris, en plein debussysme (ibid.). Soulignons toutefois que ledit essai et les mémoires de Casella datent respectivement des années 1930 et 1941 et qu'il a alors une opinion beaucoup plus tranchée à propos de l'impressionnisme, avec le recul, qu'il ne l'avait en 1909.

Après le déclenchement de la Première Guerre mondiale, Casella demeure encore quelques mois à Paris. Le 4 juin 1915, il organise un concert bénéfice au profit de la Croix Rouge italienne. Le concert se déroule à la Salle Gaveau avec le concours de Debussy $^{21}$.

20 Suite à son retour en Italie, en 1915, Casella conseille fortement aux compositeurs italiens de la jeune génération d'abandonner toute forme de vérisme et par le fait même, toute musique vocale, pour se concentrer sur la création de musique instrumentale, inspirée de la musique classique italienne.

21 Programme : D. Scarlatti, Cinque sonate pour violoncelle et piano ; Camille Saint-Saëns, Sonate pour violoncelle et piano ; Claude Debussy, Ibéria (pour deux pianos); Giacobbe Cervetto, Sonate pour violoncelle ; 
Casella et la musique italienne : vers une nationalité musicale

À son retour en Italie en 1915, Casella cherche à raviver et à moderniser la musique instrumentale italienne en puisant certes dans la grande tradition du pays, mais également en y introduisant les techniques compositionnelles européennes les plus modernes. Il insiste sur le développement d'une musique nationale classique, qui brise définitivement les stéréotypes qu'avait imposés la musique opératique du $\mathrm{XIX}^{\mathrm{e}}$ siècle, reprenant ainsi la place qu'elle occupait jadis au sein des plus grandes écoles musicales européennes. Il s'efforce d'ailleurs de faire connaître les musiques modernes européennes au public et aux compositeurs italiens, grâce aux activités de ses sociétés de concerts, soit la Società Italiana di Musica Moderna (1916-1918) et la Corporazione delle Nuove Musiche (1924-1928), fondée en collaboration avec le poète Gabriele d'Annunzio (1863-1938).

La musique de Debussy est régulièrement représentée lors de ces concerts italiens et Casella exhortera les jeunes compositeurs italiens et européens à étudier sa musique. Il insiste sur le fait que les musiques étrangères ne doivent pas être balayées du revers de la main, ces dernières constituant pour des musiciens dits nationaux une source d'inspiration incommensurable permettant d'enrichir leur style musical, et ceci vaut bien sûr pour les jeunes compositeurs italiens. Il précise son idée en prenant bien soin de définir la nationalité musicale que les compositeurs italiens doivent aspirer à atteindre : une musique non pas folklorique et populaire, mais bien une musique qui contient en elle-même les caractéristiques d'une race, d'une culture, d'un goût. Casella cite à plusieurs reprises Debussy comme l'un des modèles de cette "bonne » nationalité musicale :

Il y a deux sortes de "nationalités » en musique : la première, celle folklorique, ou populaire, la plus facilement identifiable par tous (du type Rimski-Korsakov, Albéniz, des Hongrois modernes, etc.) ; le caractère plus ou moins national de la musique conforme à ce principe varie en raison directe de la plus ou moins grande originalité et diffusion mondiale du folklore inspirateur. Il y a ensuite une autre "nationalité » : celle qui est pour ainsi dire "impondérable ", parce que constituée de mille choses occultes de race, de culture, d'atavisme, d'esthétique, de goût, de technique, etc. Celle-ci est la " nationalité » d'un Schumann ou d'un Debussy, " nationalité » que je considère de beaucoup supérieure à l'autre, parce que beaucoup plus profonde, mais qui est par ailleurs beaucoup moins caractéristique et exige de l'auditeur une connaissance infiniment plus vaste de la patrie et des affinités ethniques et intellectuelles du compositeur ${ }^{22}$.

Monteverdi, Lamento d'Ariana ; Gabriel Fauré, Quatre mélodies (s.d.). Interprètes : André Hekking, violoncelle ; Claude Debussy et Alfredo Casella, piano ; Claire Croiza, soprano.

22 "In musica vi sono due specie di "nazionalità": primo, quella folkloristica, o popolare, la più immediatamente afferrabile per tutti (tipo Rimsky-Korsakow, Albeniz, ungheresi moderni, ecc.); il carattere più o meno nazionale della musica informata a questo principio varia in ragione diretta della più o meno grande originalità e diffusione mondiale del folklore ispiratore. V'ha poi un'altra nazionalità: quella costituita da mille cause occulte di razza, di coltura, di 
Dans le numéro spécial de la Revue musicale de décembre 1920 consacré au compositeur français, Casella s'exprime sur la figure de Debussy modèle, avec un article intitulé "Debussy et la jeune école italienne "(Casella 1920, p. 213-215). Casella soutient dans cet article que le maître français a influencé la nouvelle génération de compositeurs de la péninsule de deux manières. D'abord, en pratiquant une musique " émancipatrice " et affranchie des dogmes occidentaux, c'est-à-dire une musique antidiatonique et ensuite, en ayant adopté certains principes impressionnistes, courant dont il est le principal représentant. Toutefois, loin de clore un cycle de l'évolution musicale comme l'avait fait le wagnérisme, Casella considère plutôt à cette époque que le debussysme en inaugure un nouveau. Une musique libre de tout dogme telle est, selon lui, la principale influence exercée par Debussy en Italie sur sa génération.

Or, si Casella reconnaît que Debussy a eu une influence marquante en Italie d'un point de vue harmonique ou plutôt antidiatonique (pour reprendre son expression), il nie toute adhésion possible des Italiens à l'impressionnisme français :

La nature, chez nous, est absolument antiimpressionniste. Nul brouillard dans nos paysages, nul mystère dans les lointains, mais toujours une netteté de contours fine et implacablement précise, une lumière essentiellement classique. ... Il apparaît dès lors évident que le génie italien est avant tout fait de robustesse, de plénitude, de clarté, et aussi surtout d'un grand " sens du volume » (ibid., p. 215).

Si au début des années 1920 Casella entend la musique de Debussy comme étant la réaction logique à celle de Wagner et lui confère un rôle historique de premier ordre, son impressionnisme ne constitue pas, à ses yeux, une solution au renouvellement de la musique nationale italienne, d'autant qu'elle serait incompatible avec le «bon goût » italien. S'il croit fermement que la grande majorité des influences étrangères soit salutaire à l'art musical italien, il n'en reste pas moins que quelquesunes, dont l'impressionnisme, sont à proscrire. Son article "Impressionismo e anti-medesimo ${ }^{23}$ " datant de 1918 faisait déjà état des effets néfastes que la "fièvre debussyste ", tout comme la "fièvre wagnérienne " qui la précédait, pouvait avoir sur la musique moderne italienne. Le debussysme se préoccuperait trop du plaisir auditif et négligerait ainsi des facteurs primordiaux pour la musique italienne : la forme, l'ossature rythmique et l'unité mélodique (Casella 1918, p. 5) ${ }^{24}$. Également, Casella reproche à l'esthétique debussyste d'être docilement soumise aux facteurs extramusicaux que sont le symbolisme poétique et l'impressionnisme pictural. Il ne niera pas

atavismo, di estetica, di gusto, di tecnica, ecc. Questa è la nazionalità di un Schumann o di un Debussy, "nazionalità" che io reputo di gran lunga superiore all'altra, perché assai più profonda, ma che è d'altronde molto meno caratteristica, ed esige dall'uditore una conoscenza infinitamente più vasta della patria e delle affinità etniche ed intellettuali del compositore» (Casella 1916, p. 2).

23 «Impressionnisme et anti-impressionnisme».

24 Notons que ces reproches formulés contre l'art debussyste ne se limitent pas aux Italiens, mais se généralisent partout en Europe à cette époque et même en France, avec Le coq et l'arlequin de Jean Cocteau (1979). 
l'importance de l'œuvre de Debussy et en soulignera même certains aspects essentiels comme l'évolution de la modalité, de l'harmonie tonale et de la rythmique, pour lesquels les compositeurs italiens lui sont redevables. Il affirme cependant :

L'Italien n'est pas enclin à l'impressionnisme et ce pour mille raisons géographiques, ethniques et culturelles. D'abord, la nature de notre pays, le classicisme et la transparence de sa lumière, l'absence presque totale de nébulosité et de mystère de nos paysages, ne sont pas favorables à la vision impressionniste. En outre, l'Italien possède trop de sens plastique pour se complaire en un art dont la principale caractéristique est justement la négation du dessin et de la forme ${ }^{25}$.

Casella définit la nouvelle musique italienne comme l'antipode du debussysme, par son dynamisme rythmique, sa construction plastique, sa robustesse strophique et sa linéarité claire. Ironiquement, il affirme justement, lors de sa période française, que chez Debussy c'est cette clarté, cette netteté de forme, cette perfection de réalisations qui l'avaient charmé (Casella, « Saint-Saëns et les jeunes »).

\section{DEBUSSY WAGNÉRIEN OU ANTI-WAGNÉRIEN?}

La réception casellienne de Debussy comporte un dernier aspect, non moins important. Il s'agit sans doute de la question qui divise le plus l'opinion générale face à la musique debussyste, tant en France qu'en Italie, c'est-à-dire celle qui consiste à faire du compositeur un réactionnaire ou un successeur à la musique de Wagner. Casella se penchera lui-même sur cette question, arrivant à diverses conclusions selon les époques. Répétons que Casella considérait l'œuvre de Debussy comme une nouvelle étape dans l'histoire de la musique, comme une rupture avec l'art wagnérien. Il affirmait au cours des années 1910 et 1920 que le compositeur français avait formé son étonnante personnalité en niant Wagner de toutes ses forces (Casella 1922), et qu'il était parvenu à vaincre la tyrannie du chromatisme wagnérien par le raffinement diaphane de son système tonal (Casella 1913, p. 41).

Dans un article de 1923, intitulé "Problemi sonori odierni ${ }^{26}$ ", Casella parle même de la musique de Debussy comme d'un véritable miracle survenu après cette vieille forteresse dogmatique du wagnérisme (Casella 2001, p. 31). Cependant, dans les années 1930, la vision casellienne se métamorphose et il se déclare profondément antiromantique. Il soutient que les compositeurs italiens de la jeune génération doivent faire table-rase de la période romantique pour ne se concentrer que sur l'étude des compositeurs baroques et classiques. En tant qu'antiromantique, il répudie l'ul-

25 "L'Italiano non è incline all'impressionismo, e ciò per mille ragioni geografiche, etniche e culturali. Anzitutto, la natura del nostro paese, il classicismo e la trasparenza della sua luce, la assenza quasi totale di nebulosità e di mistero dei nostri paesaggi, non sono favorevoli alla visione impressionistica. Inoltre, 'italiano possiede troppo senso plastico per compiacersi in un'arte di cui la principale caratteristica è appunto la negazione del disegno e della forma » (Casella 1918, p. 5).

26 «Problèmes sonores d'aujourd'hui ». 
tra-sentimentalisme, la décadence (Casella 1934, p. 6) et les excès chromatiques de la musique de cette période.

Il rejette également l'art wagnérien qu'il conçoit comme le représentant par excellence de ces excès et, enfin, le debussysme, qu'il ne voit plus comme une nouvelle ère musicale, mais plutôt comme l'héritier des excès wagnériens. Debussy devient dans ce contexte le compositeur qui, malgré le souffle rénovateur qu'il avait insufflé à la musique, clôt la période romantique et dont l'esthétique est déjà surannée. Notons que cette conception négative du debussysme s'inscrit dans une tendance qui compare l'œuvre du compositeur français à une maladie infectieuse, la " debussyte ", dont les étranges symptômes seraient le ramollissement des membres, la pâleur soudaine du visage et le voilage des yeux (Setaccioli 2010)!

\section{CONCLUSION}

Il ne fait pas de doute que la métamorphose de la réception casellienne de l'œuvre de Debussy soit corollaire à la montée du fascisme en Italie. Souvenons-nous de ses encouragements lancés aux jeunes Italiens à étudier les musiques européennes. En 1935, Casella tient un tout autre discours. Si les compositeurs italiens ont jadis été influencés par les musiques allemandes, russes et françaises, il estime désormais que le Fascisme a insufflé aux Italiens cette unité morale qu'ils ne connaissaient pas avant, permettant aux artistes et aux musiciens d'atteindre une certaine indépendance de pensée et de forme, face aux musiques étrangères, que la génération antérieure ignorait totalement (Casella 1935, p. 162).

L'attitude de Casella a ceci de particulier qu'il sera d'abord un grand admirateur de Debussy. Cette admiration se traduit par le caméléonisme stylistique des œuvres déjà citées. Une admiration qui se transforme par la suite en un détachement fortement affirmé dans ses déclarations orales et écrites. Cependant, s'il rejette l'esthétique impressionniste avec vigueur dans ses écrits, il en va autrement dans certaines de ses œuvres musicales, postérieures à sa période française, qui contiennent encore plusieurs références debussystes (Minardi 1994, p. 252) ${ }^{27}$.

Casella a sans doute été, en Italie, le plus sévère critique de Debussy, mais également, sans contredit, son plus fervent admirateur. Le compositeur français est en effet pour lui un modèle parfait de ce qu'il décrit comme la nationalité en musique. Cependant, il exhortera toujours les compositeurs italiens de la jeune génération à ne pas suivre l'exemple de l'impressionnisme, musique susceptible, selon lui, de menacer l'intégrité de l'identité italienne. Il voyait jadis Debussy comme la lumière d'un phare qui montrait la voie à suivre pour la nouvelle musique. Cependant, avec les années, cette lumière nouvelle s'est métamorphosée en une faible lueur, en une mode éphémère déjà désuète, une mode à éviter. 


\section{BIBLIOGRAPHIE}

Audoin-Rouzeau, Stéphane, et al. (dir.) (2009), La grande guerre des musiciens, Lyon, Symétrie.

Buch, Esteban (2009), "Composer pendant la guerre, composer avec la guerre ", dans Stéphane Audoin-Rouzeau et al. (dir.), La grande guerre des musiciens, Lyon, Symétrie, p. 135-160.

Calabretto, Roberto (2001), « Casella, Malipiero e Pizzetti. Tre modi diversi di rapportarsi alla musica da film », Carte di Cinema, no 8 (été/hiver), p. 94.

Casella, Alfredo (1910), "La Huitième Symphonie de Gustav Mahler. Notes sur l'Exécution ", Le monde musical, p. 259-260.

Casella, Alfredo (1910) « Mise au point. À propos des modifications apportées par M. G. Mahler dans l'instrumentation des Symphonies de Beethoven ", Le monde musical (30 janvier), p. 22-24.

Casella, Alfredo (1910), "Gustav Mahler et sa Deuxième Symphonie », Revue musicale SIM (15 avril), p. 238-250.

Casella, Alfredo (1911), "La Cinquième Symphonie de Mahler », Le monde musical (30 janvier), p. 26.

Casella, Alfredo (c1910), « Saint-Saëns et les jeunes », Le guide du concert, numéro hors-série.

Casella, Alfredo (1911), " Gustav Mahler », Le guide du concert, p. 425-426.

Casella, Alfredo (1911), "Gustav Mahler », Le monde musical, p. 154.

Casella, Alfredo (1913), «Giannotto Bastianelli. La crisi musicale europea » [ Giannotto Bastianelli. La crise musicale européenne »], Revue musicale SIM (mars), p. 41.

Casella, Alfredo (1914), « Ce qu'est la musique polyharmonique », Montjoie! (juin).

Casella, Alfredo (1914), "Le Sacre du printemps aux Concerts Monteux », L'homme libre (6 avril), p. 2.

Casella, Alfredo (1915), "L'evoluzione armonica modernissima », La Riforma musicale (7 févier), p. 1.

Casella, Alfredo (1916), «Arte e patria », Musica (25 janvier), p. 2.

Casella, Alfredo (1918), "Impressionnismo e antimedesimo » [" Impressionnisme et antiimpressionnisme »], Ars Nova, vol. 2, no 4 (mars), p. 1-5.

Casella, Alfredo (1920), "Debussy et la jeune école italienne », Revue musicale (décembre), p. 213215.

Casella, Alfredo (1922), Conférence inédite donnée à NY.

Casella, Alfredo (1922), « Why I write as I do » [« Pourquoi j’écris comme je le fais »], The Musical Courier (2 mars), p. 34-35.

Casella, Alfredo (1926), « Harmonie, contrepoint, etc. », Courrier musical (avril), p. 185-186.

Casella, Alfredo (1930), "Casella veduto da... se stesso » [ Casella vu par... lui-même »], Musica d’Oggi (décembre), p. 495-500.

Casella, Alfredo (1934), « The Decline of Modernity » [ "Le déclin de la modernité »], Musical Courier (24 mars), p. 6.

Casella, Alfredo (1935), «Problemi della musica contemporanea italiana » [« Problèmes de la musique contemporéenne italienne »], La rassegna musicale italiana (mai-juin), p. 162.

Casella, Alfredo (1941), I Segreti della giara [" Les secrets de la jarre »], Florence, Sansoni.

Casella, Alfredo (2001), 21+26, Florence, Leo S. Olschki.

Cocteau, Jean ([1918]1979), Le coq et l'arlequin, Paris, Stock.

Minardi, Gian Paolo (1994), "À la manière de... ", dans Giovanni Morelli (dir.), Alfredo Casella negli anni di apprendistato a Parigi, Florence, Leo S. Olschki, p. 249-257. 
Morelli, Giovanni (1994), Alfredo Casella negli anni di apprendistato a Parigi, Florence, Leo S. Olschki.

Setaccioli, Giacomo (2010), Debussy è un innovatore? [ Debussy est-il un innovateur ? ], Whitefish, Montana, Kessinger Publishing.

Spampinato, Francesco (2011), Debussy, poète des eaux. Métaphorisation et corporéité dans l'expérience musicale, Paris, L'Harmattan.

Watkins, Glenn (2003), Proof Through the Night. Music and the Great War, Berkeley, University of California Press. 